\section{Demonstration of the Epstein-Barr genome by the polymerase chain reaction and in situ hybridisation in a patient with viral pericarditis}

\author{
Toru Satoh, Masaru Kojima, Kohichi Ohshima
}

\section{Department of Cardiology, Ashikaga Red Cross Hospital, Ashikagashi, Tochigi Prefecture, Japan T Satoh \\ Department of Pathology, Ashikaga Red Cross Hospital, Ashikagashi, Tochigi Prefecture, Japan M Kojima \\ First Department of Pathology, School of Medicine, Fukuoka University, Fukuoka Prefecture, Japan K Ohshima \\ Correspondence to Dr Toru Satoh Department of Cardiology, Kawasaki Medical School, 577 Matsushima, Kurashiki-city, Okayama, 701-01 Japan.}

\begin{abstract}
A 42 year old man admitted with effusiveconstrictive pericarditis had diastolic dysfunction and pericardial thickening. Pericardiectomy was performed because of uncontrolled heart failure. The Epstein-Barr viral IgG antibody titre was exceptionally high. The EB viral genome was demonstrated in the resected pericardium by polymerase chain reaction and in situ hybridisation. EB viral pericarditis was diagnosed.
\end{abstract}

(Br Heart F 1993;69:563-564)

Both Coxsackie B virus and Epstein-Barr (EB) virus are known to cause pericarditis. ${ }^{1}$ We report a patient with effusive-constrictive pericarditis and a high EB virus antibody titre in whom EB viral pericarditis was diagnosed when the polymerase chain reaction (PCR) and in situ hybridisation showed the EB virus genome in a small sample of pericardium.

\section{Case report}

A 42 year old man was admitted with generalised oedema and dyspnoea. He was a heavy

\section{4}
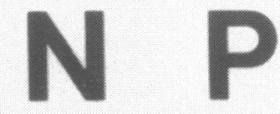

Figure $1 P C R$ applied to the resected pericardium ${ }^{5}(P$ represents the positive and $N$ the negative control for $E B$ virus. The numbers $1,2,3,4$ represent the magnitude of dilution. The "hot spot" appears in region 1 in this patient). Specific primers corresponding to the $B a m$ HIW region of $E B$ virus were synthesised. Amplification was achieved with a GeneAmp DNA amplification reagent kit and DNA Thermal Cycler Perkin-Elmer Cetus, Norwalk, CT). After 40 cycles of PCR amplification, $1 / 100$ of the reaction mixture was analysed by the dot-blot method with a ${ }^{32}$ P-labeled oligonucleotide probe. drinker with a history of alcoholic liver dysfunction. On examination he was found to be mildly dyspnoeic. An increase in jugular venous pressure, decreased respiratory sounds over both the lung bases, an enlarged and tender liver, and pretibial oedema were noted. An $x$ ray film of the chest showed bilateral pleural effusions. Echocardiography showed massive pericardial effusion and pericardial thickening. A computed tomographic scan of the thorax and abdomen confirmed pericardial thickening and pleural and abdominal effusions. Right-sided cardiac catheterisation showed a right atrial pressure of $17 \mathrm{~mm} \mathrm{Hg}$ (mean) with a steep " $y$ " wave descent and a right ventricular pressure of $37 / 17 \mathrm{~mm} \mathrm{Hg}$ with a "dip and plateau" pattern, a pulmonary arterial pressure of $33 / 19 \mathrm{~mm} \mathrm{Hg}$, a mean pulmonary arterial wedge pressure of $20 \mathrm{~mm}$ $\mathrm{Hg}$ (with similarly raised diastolic pressures in all four heart chambers), and a low cardiac index $(2 \cdot 3)$, indicating ventricular diastolic abnormality. The low cardiac index and pericardial thickening with pericardial effusion led to a diagnosis of effusive-constrictive pericarditis. Only the antibody titres to EB virus were exceptionally high. The IgG antibody titre to the viral capsid antigen (EBVCA IgG) was 5120 , and the antibody titre to the viral nuclear antigen (EBNA) was 160. The tuberculin test was positive. These findings suggested that the patient had EB viral or tuberculous pericarditis.

Though the pericardial effusion disappeared after treatment with pericardial drainage, diuretics, and antitubercular agents, the right atrial pressure remained raised and he had a pericardiectomy in May 1991. Microscopic examination of a specimen of resected pericardium showed an inflammatory reaction. PCR (fig 1) and in situ hybridisation (fig 2) showed the presence of EB viral genome in the specimen.

\section{Discussion}

EB viral antibody titres as high as we found in this patient are rare in adults. The high EBVCA IgG and EBNA titres suggested sustained infection or reactivation of $E B$ virus.

Immunological suppression can reactivate the EB virus-as it was reported to do in renal transplantation patients in whom lymphoma associated with $\mathrm{EB}$ virus developed. ${ }^{2} \mathrm{~A}$ patient 


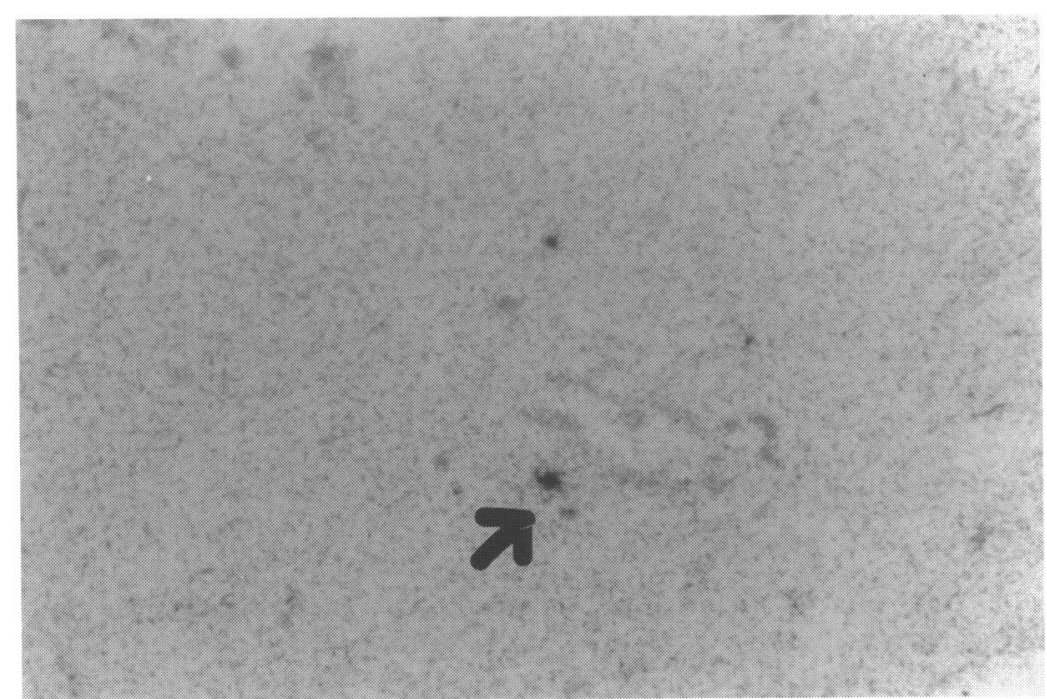

Figure 2 In situ hybridisation studies of the resected pericardium ${ }^{5}$ (the EB virus genome is at the tip of the arrow). The EB virus DNA probe consisted of the Bam HIV region of $E B$ virus DNA (internal repair 1), which had been radiolabelled with $a-{ }^{35}-S d C T P$ by the random hexamer primer technique. Tissue sections $(3 \mu \mathrm{m})$ were deparaffinised with

xylene. The sections were digested and acetylated. Then a $50 \mu l$ sample of the hybridisation mixture, containing the appropriate radiolabelled probe mixed in prehybridisation solution, was then hybridised for $16 \mathrm{~h}$ at $42^{\circ} \mathrm{C}$. After hybridisation, autoradiographic exposure was continued for 14-21 days. After the emulsion was developed the sections were counterstained with haematoxylin.

with impaired all-mediated immunity is susceptible to EB virus infection: this was not the case in our patient, who had a positive tuberculin test. In our patient alcoholic liver injury may have suppressed immunity to pathogens. ${ }^{3}$

In our patient with $\mathrm{EB}$ viral pericarditis the
EB viral genome was demonstrated by PCR, which may not be specific because in small specimens the genes are multiplied several dozen times. However, the presence of the virus was confirmed by in situ hybridisation. There have been several reports of detection of the EB viral genome in human specimens-for example, in tonsillar tissue in patients with infectious mononucleosis, ${ }^{4}$ in lymphocytes from patients with malignant lymphoma, and in craniopharyngioma tissue. We believe that this is the first report of the demonstration of EB viral genome in the resected pericardium from a patient with viral pericarditis. We expect that more cases of pericarditis and myocarditis related to or caused by viruses will be diagnosed by these immunobiological techniques.

1 Webster BH. Cardiac complications of infectious mononucleosis. A review of the literature and report of five cases. Am $\mathcal{F}$ Med Sci 1947;234:62-9.

2 Hanto DW, Frizzera G, Gail-Peczalska KJ, Sakamoto K, Purtilo DT, Balfour $\mathrm{HH}$, et al. Epstein-Barr virusinduced B-cell lymphoma after renal transplantation. $N$ Engl ₹ Med 1982;306:913-8.

3 Bagasra O, Kajdacsy-Balla A, Lischner HW. Effects of alcohol ingestion on in vitro susceptibility of peripheral blood mononuclear cells to infection with HIV and of selected T-cell functions. Alcohol Clin Exp Res 1989;13:636-43.

4 Niedobitek G, Hamilton-Dutoit S, Herbst H, Finn T, Vetner M, Pallesen G, et al. Identification of EpsteinBarr virus-infected cells in tonsils of acute infectious mononucleosis by in situ hybridization. Hum Pathol 1989;20:796-9.

5 Ohshima K, Kikuchi M, Eguchi F, Masuda Y, Sumiyoshi $\mathrm{Y}$, Mohtai $\mathrm{H}$, et al. Analysis of Epstein-Barr viral genomes in lymphoid malignancy using southern blotting, polymerase chain reaction and in situ hybridiza tion. Virchows Arch [B] 1990;59:383-90. 